\title{
Detección de aneuploidías del cromosoma 17 y deleción del gen TP53 en una amplia variedad de tumores sólidos mediante hibridación in situ fluorescente bicolor
}

\author{
Juan Carlos Herrera¹, Luis Fernando Isaza², José Luis Ramírez¹, Gonzalo Vásquez¹, \\ Carlos Mario Muñetón ${ }^{1}$ \\ 1 Unidad de Genética Médica, Facultad de Medicina, Universidad de Antioquia, Medellín, Colombia
2 Departamento de Cirugía, Hospital Universitario San Vicente de Paúl, Facultad de Medicina, Universidad de
Antioquia, Medellín, Colombia
}

Introducción. EI TP53 es un gen supresor de tumores localizado en la región cromosómica 17p13.1; controla el ciclo celular y se encuentra alterado en cerca de $50 \%$ de todas las neoplasias.

Objetivos. Determinar las aneuploidías del cromosoma 17 y la deleción en el locus 17p13.1 del gen TP53, en diversos tumores sólidos primarios utilizando la técnica FISH-bicolor.

Materiales y métodos. Se analizaron 38 muestras de diversos tipos de tumores sólidos primarios. Todas las muestras se disociaron mecánica y enzimáticamente con colagenasa al $0,2 \%$, antes de la obtención de los núcleos interfásicos. La técnica de FISH-bicolor se realizó en núcleos interfásicos, mediante sondas marcadas directamente con fluorocromos para el centrómero del cromosoma 17 (CEP 17; señal verde; VYSIS) y para el locus específico del gen TP53 (LSI 17p13.1; señal naranja; VYSIS).

Resultados. Se encontró que 63\% (24/38) de las muestras tenían aneuploidías del cromosoma 17. La monosomía fue la aneuploidía más frecuente (75\%; 18/24), seguida de la trisomía (17\%; 4/24); la nulisomía y la tetrasomía fueron menos frecuentes. El 89,5\% (34/38) de los casos presentaron deleción del gen TP53. Sólo cuatro casos fueron normales para el número de copias del cromosoma 17 y del gen TP53. El estudio histopatológico mostró que la mayoría de las muestras eran tumores malignos.

Conclusiones. La aneuploidía del cromosoma 17 y la deleción en el locus 17p13.1 del gen TP53 son alteraciones muy frecuentes en los tumores sólidos. La técnica FISH-bicolor permite detectar simultáneamente alteraciones cromosómicas numéricas y estructurales en núcleos interfásicos.

Palabras clave: aneuploidía, deleción cromosómica, gen Tp53, hibridación fluorescente in situ, neoplasias, inestabilidad cromosómica, heterogeneidad genética.

Detection of chromosome 17 aneuplody and TP53 gene deletion in a broad variety of solid tumors by dual-color fluorescence in situ hybridization (FISH)

Introduction. TP53 is a tumor suppressor gene located on chromosome 17p13.1. This gene is essential for the control of cell cycle and has been found altered in about $50 \%$ of all tumor types.

Objective. The presence of aneuploidy of chromosome 17 and TP53 gene deletion at 17p13.1 locus was determined in primary solid tumors using the dual-color FISH (fluorescence in situ hybridization).

Materials and methods. Thirty-eight samples consisted of several types of primary solid tumors. All samples were mechanically and enzymatically disaggregated with $0.2 \%$ collagenase prior to obtaining interphase nuclei. The dual-color FISH was performed using direct fluorescent labeling probes for the chromosome 17 centromere (green signal) and for the TP53 gene locus-specific (orange signal).

Results. Characteristic aneuploidy on chromosome 17 was found in $63 \%(24 / 38)$ of the samples. Monosomy occurred most frequently $(75 \%, 18 / 24)$, followed by trisomy $(17 \%, 4 / 24)$; nullisomy and tetrasomy were less frequent. TP53 gene deletion was found in $89.5 \%(34 / 38)$ of cases. Only four tumors were normal for copy number of chromosome 17 and TP53 gene. The histopathologic study showed that most of the samples were malignant tumors.

Conclusions. Aneuploidy of chromosome 17 and deletion at 17p13.1 locus of TP53 gene were genetic alterations found to be very frequent in solid tumors. The dual-color FISH was able to detect both numerical and structural chromosomal abnormalities in interphase nuclei.

Key words: Aneuploidy, chromosome deletion; genes, p53; in situ hybridization, fluorescence; neoplasms, chromosomal instability, genetic heterogeneity. 
El cáncer se origina por la acumulación de diversas alteraciones genéticas en genes supresores de tumores, protooncogenes o genes de reparación (1). La mayoría de los tumores sólidos humanos presenta una amplia variedad de alteraciones génicas y de desequilibrios cromosómicos (ganancias y pérdidas) (2). Además, la inestabilidad cromosómica es una característica común de los tumores sólidos.

Desde hace décadas se ha propuesto la teoría de la aneuploidía como causa de la carcinogénesis, así como de la inestabilidad cromosómica (3). Numerosos estudios informan la presencia de aneuploidías en casi todos los tumores sólidos humanos que se han analizado $(2,4)$. Los recientes trabajos realizados por Duesberg et al. (5) están dirigidos a corroborar la anterior teoría y en ellos se establece que la inestabilidad cromosómica de las células tumorales es proporcional al grado de la aneuploidía o del desequilibrio cromosómico (6). Por esta vía, las alteraciones cromosómicas afectan la expresión de genes clave, como son los del control del ciclo celular, de la estabilidad genómica, de la angiogénesis, de la apoptosis y de la reparación, entre muchos otros (4).

Por otra parte, se considera que las alteraciones en el gen TP53 juegan un papel importante en el desarrollo de diversas neoplasias. EI TP53 es un gen supresor de tumores, localizado en el brazo corto del cromosoma 17p13.1; codifica para una fosfoproteína nuclear de $53 \mathrm{kDa}$, que actúa como un factor de transcripción e interviene en múltiples funciones celulares, especialmente, en el control del ciclo celular, la apoptosis, la respuesta al daño y la reparación del $\operatorname{ADN}(7,8)$; por lo anterior, también se le conoce como "gen guardián del genoma humano". El gen TP53 se encuentra alterado en cerca de $50 \%$ de todos los cánceres estudiados y se ha observado más frecuentemente alterado en los tumores sólidos que en las leucemias y linfomas (7). Además, el TP53 se propone como un marcador genético útil para el diagnóstico y el pronóstico de diversos tipos de tumores sólidos (9).

Las mutaciones en el gen TP53 y las deleciones en la región $17 p$ están entre las alteraciones más

\footnotetext{
Correspondencia:

Carlos Mario Muñetón, Unidad de Genética Médica, Facultad de Medicina, Universidad de Antioquia, Carrera 51D № 62-29, Medellín, Colombia

Teléfono: (054) 219 6930; fax: (054) 2196932

cmuneton@quimbaya.udea.edu.co

Recibido: 17/06/09; aceptado:27/04/10
}

comunes encontradas en tumores sólidos primarios de diferente origen histológico $(4,9)$; ambos tipos de alteraciones inducen a la inestabilidad genómica en las células transformadas. Asimismo, se ha observado que la inactivación o pérdida del gen TP53 se asocia con un mal pronóstico y estados avanzados del cáncer, así como con la progresión de diversas neoplasias (10), lo cual sugiere que la inactivación de este gen o la deleción de la región 17p tiene una gran importancia en la carcinogénesis, tal como se ha observado en el cáncer de mama, estómago, hígado y colon, y en tumores de cabeza $(8,9)$.

En trabajos previos, encontramos una gran frecuencia de aneuploidías del cromosoma 17 y de deleción del gen TP53en tumores gastrointestinales (11); pero, por el contrario, encontramos unas bajas frecuencias de estas mismas alteraciones en neoplasias hematológicas (12).

De otro lado, el desarrollo de la técnica de la hibridación fluorescente in situ (fluorescence in situ hybridization, FISH) permitió un gran avance en los análisis citogenéticos de tejidos tumorales, puesto que posibilitó detectar simultáneamente aneuploidías, deleciones, translocaciones y amplificaciones génicas, entre otros reordenamientos cromosómicos, en núcleos interfásicos, sin tener que establecer cultivos celulares (13). Por el contraio, con la citogenética convencional el análisis cromosómico de células tumorales tiene varios inconvenientes, como son el bajo índice mitótico, un bandeo deficiente y la contaminación con microorganismos; lo anterior impide el éxito en los cultivos celulares y en la identificación de las alteraciones cromosómicas (14).

Otra de las grandes ventajas que se tiene con la FISH en la citogenética del cáncer, es la posibilidad de realizar estudios retrospectivos de tejidos embebidos en bloques de parafina o congelados. Por lo anterior, la FISH permite realizar un análisis citogenético molecular rápido, con gran especificidad y sensibilidad en células de tumores sólidos y hematopoyéticos.

En Colombia se han informado pocos estudios sobre la detección de estas alteraciones en tumores sólidos y menos aún empleando la técnica de la FISH-bicolor. El objetivo del presente trabajo fue el de evaluar las aneuploidías del cromosoma 17 y la deleción del locus 17p13.1 del gen TP53, utilizando la técnica FISH-bicolor, en muestras de individuos con diferentes tipos de tumores sólidos. 


\section{Materiales y métodos}

\section{Pacientes}

Se realizó un estudio descriptivo, de tipo prospectivo; se evaluaron 38 muestras provenientes de pacientes con diagnóstico de diversos tipos de neoplasias; las muestras de los tejidos fueron obtenidas por los médicos especialistas, mediante procedimientos de resección quirúrgica o biopsia del tumor primario, practicados en los Servicios de Cirugía y Endoscopia del Hospital Universitario San Vicente de Paúl de Medellín.

Los pacientes no habían recibido quimioterapia ni radioterapia antes de la toma de la muestra. Las muestras provenían de 16 mujeres y 22 hombres procedentes del departamento de Antioquia, con un promedio de edad de 52 años y un rango entre 23 y 78 años.

Un fragmento de cada muestra de tejido tumoral se envió al Laboratorio de la Unidad de Genética Médica, de la Facultad de Medicina, de la Universidad de Antioquia, en donde se practicaron los estudios de FISH; mientras que otro fragmento de la misma muestra del tejido se envió al Departamento de Patología de la Universidad de Antioquia, en donde se hizo el estudio histopatológico. El resultado de este estudio se obtuvo luego de la revisión de la historia clínica de cada paciente, con el fin de corroborar el diagnóstico de las neoplasias de las muestras evaluadas. También se obtuvo información adicional como: edad, sexo, procedencia y antecedentes familiares.

Como control se incluyeron cinco muestras de sangre periférica pertenecientes a cinco individuos sanos sin antecedentes de cáncer.

\section{Consideraciones éticas}

Este trabajo fue producto de un proyecto de investigación aprobado por el Comité de Ética de la Universidad de Antioquia. Cada paciente participó voluntariamente en el estudio y se obtuvo su consentimiento informado escrito antes de ingresar al estudio.

\section{Procesamiento de las muestras para obtener núcleos interfásicos}

Las muestras de los tumores sólidos y de los controles se procesaron para obtener núcleos interfásicos. Los tejidos tumorales fueron recolectados y transportados en un frasco plástico con medio de transporte (RPMI1640 Sigma; con suplemento de antibióticos y antimicóticos); los tejidos se lavaron dos veces con
PBS, luego se cortaron finamente y se disociaron enzimáticamente con colagenasa de tipo I (Sigma) al $0,2 \%$ y se incubaron a $37^{\circ} \mathrm{C}$ entre 1 y 16 horas.

Posteriormente, se centrifugaron a 800 rpm por 10 minutos; el botón celular se resuspendió en una solución hipotónica de $\mathrm{KCl}$ al 0,075 M y se incubó a $37^{\circ} \mathrm{C}$ por 15 minutos. Luego, la suspensión celular se fijó con metanol/ácido acético frío (3:1 volumen/ volumen) y se hicieron tres lavados consecutivos.

Finalmente, para cada muestra la suspensión celular se goteó sobre tres portaobjetos como mínimo. Las placas con los extendidos de núcleos interfásicos se almacenaron en un congelador a $-20^{\circ} \mathrm{C}$, hasta el momento de realizar la técnica de la FISH-bicolor.

Para el grupo control, a cada individuo se le tomó una muestra de $5 \mathrm{ml}$ de sangre periférica en un tubo con heparina. Posteriormente, los tubos se centrifugaron y el botón celular obtenido se resuspendió en $\mathrm{KCl}$ al $0,075 \mathrm{M}$; los núcleos interfásicos de estas muestras se obtuvieron siguiendo el mismo procedimiento que se mencionó anteriormente; finalmente, se gotearon cinco láminas portaobjetos por cada muestra.

\section{FISH bicolor}

La hibridación fluorescente in situ se realizó sobre las láminas de vidrio con núcleos interfásicos obtenidos de las muestras tumorales y de las muestras control, utilizando sondas marcadas directamente con diferentes fluorocromos para el centrómero del cromosoma 17 (CEP 17; señal verde; VYSIS) y para el locus específico del gen TP53 (LSI 17p13.1;señal naranja;VYSIS).

La hibridación y detección de las señales de fluorescencia se hicieron siguiendo las instrucciones y recomendaciones del proveedor comercial (Vysis-Abbott). Las placas con núcleos interfásicos se desnaturalizaron en formamida al $70 \%$ a $73^{\circ} \mathrm{C}$ por 5 minutos. Después, las placas se deshidrataron en una serie de etanoles fríos a $70 \%$, $85 \%$ y $100 \%$. En cada placa se adicionaron $10 \mu \mathrm{l}$ de cada una de las sondas CEP 17 y LSI TP53 en el área de hibridación previamente seleccionada, sobre la cual se puso un cubreobjetos de $22 \mathrm{~mm}$ x $22 \mathrm{~mm}$; la hibridación se llevó a cabo dentro de una cámara húmeda en incubación a $37^{\circ} \mathrm{C}$ por 16 horas. Luego, se hicieron dos lavados posteriores a la hibridación.

Finalmente, las placas con núcleos interfásicos se colorearon con DAPI. Las señales de fluorescencia 
se observaron en un microscopio de epifluorescencia (Axyoskop 2 plus Zeiss, Alemania) dotado con un filtro triple simultáneo (DAPI/ORANGE/ GREEN) y con una cámara CCD AxioCam MRc (Zeiss). Un profesional capacitado en la técnica de FISH fue el encargado de realizar el análisis y conteo de los núcleos con señales de hibridación en un total de 100 núcleos interfásicos por caso.

La distribución de las señales observadas en las muestras analizadas se registró en tablas como aparece en el cuadro 1. Los núcleos superpuestos o con señales de hibridación difusas se excluyeron del análisis. Para la interpretación de los resultados de las señales de fluorescencia se establece que, en los casos normales (controles), el patrón de hibridación es de dos señales de color verde (cromosoma 17) y dos señales de color naranja (locus 17p13.1 del gen TP53), para un total de cuatro señales de hibridación (2V2N).

\section{Análisis de la FISH bicolor}

Inicialmente se hicieron análisis de las muestras de control (sangre periférica y tejido normal) (no se muestran los datos) en 1.000 núcleos interfásicos, se estimó el número promedio de núcleos con señales de hibridación para el cromosoma 17 y para el gen TP53.

De acuerdo con los anteriores resultados, se establecieron los siguientes puntos de corte para el análisis de la FISH bicolor: para aneuploidía, más de $9 \%$ de los núcleos con un número menor o mayor de dos señales de hibridación para el centrómero del cromosoma 17, es decir que la muestra se consideró normal (disómica) con un porcentaje mayor o igual a $91 \%$ de los núcleos con dos señales para el centrómero del cromosoma 17, mientras que, para la nulisomía, la monosomía y la trisomía, el punto de corte fue del $1 \%, 7 \%$ y $4 \%$, respectivamente. No se observó tetrasomía en los núcleos normales; por lo tanto, se definió arbitrariamente un porcentaje menor de $1 \%$ como punto de corte.

En todos los casos, los puntos de corte se calcularon con dos desviaciones estándar. En los casos en que se detectaron subpoblaciones de núcleos con clones heterogéneos (mosómicos/ disómicos/trisómicos/tetrasómicos) en el mismo tejido, el resultado final se estableció con base en el clon mayor, es decir, el que presentó el mayor porcentaje.

Por otro lado, la deleción en el locus 17p.13.1 del gen TP53 se determinó dividiendo el total del número observado de señales de hibridación del gen, por el total del número de señales del centrómero del cromosoma 17 (total de señales LSI TP53/total de señales CEP 17). De acuerdo con los cálculos previos realizados en las muestras de control, se definió que una muestra tenía deleción del gen TP53 cuando se obtenía un valor de 0,94 o menor. Este punto de corte se calculó con base en dos desviaciones estándar.

\section{Resultados}

En el cuadro 1 se presenta la información general de las 38 muestras procedentes de individuos con diferentes tipos de tumores sólidos; se incluye la edad, el sexo, la localización del tumor primario, el diagnóstico histopatológico y los resultados obtenidos con la FISH bicolor en núcleos interfásicos.

De las 38 muestras de tumores sólidos evaluados, se encontró que 14 (37\%) procedían de individuos menores de 40 años edad. De esta población sobresalen cuatro pacientes jóvenes entre los 23 y 25 años (casos 7, 15, 16 y 36) con tumores malignos.

El estudio histopatológico mostró que la mayoría de las muestras de los tumores sólidos analizadas (31/38) eran malignos y, además, se encontró una amplia variedad histopatológica en las muestras. Entre los tumores malignos, los más predominantes fueron los adenocarcinomas (12/38).

En este estudio se encontró que $63 \%$ (24/38) de los tumores sólidos estudiados tenían aneuploidías del cromosoma 17, mientras que $37 \%$ (14/38) eran normales para el número de copias del cromosoma 17. La monosomía fue la aneuploidía más frecuente (75\%; 18/24), seguida de la trisomía, que se detectó en $17 \%(4 / 24)$. Se observaron pocos casos con nulisomía y tetrasomía (4\%) (figura 1).

En el grupo de los casos se obtuvo un promedio de $80 \%$ para las dos copias del cromosoma 17 ; este valor fue muy inferior al promedio obtenido (95\%) en el grupo control. En la mayoría de las muestras analizadas con la FISH bicolor, se detectó la presencia simultánea de subpoblaciones de núcleos con clones heterogéneos: disómicos, monosómicos, trisómicos y, en menor proporción, tetrasómicos (cuadro 1).

De todas las muestras examinadas, el caso 5 (sarcoma de tejidos blandos) fue el que mostró el porcentaje más bajo (5\%) de núcleos con dos señales de hibridación para el cromosoma 17 y, a su vez, 
Cuadro 1. Información de la edad, sexo, diagnóstico histopatológico y resultado de la FISH bicolor en las 38 muestras de tumores sólidos evaluados.

\begin{tabular}{|c|c|c|c|c|c|c|c|c|c|c|c|c|c|}
\hline \multirow{3}{*}{ Caso } & \multirow{3}{*}{$\begin{array}{l}\text { Sexo/ } \\
\text { edad }\end{array}$} & \multirow{3}{*}{ Diagnóstico histopatológico } & \multicolumn{10}{|c|}{ Número de señales } & \multirow{3}{*}{$\begin{array}{c}\text { Deleción } \\
\text { (TP53/Cr17) } \\
\leq 0,94\end{array}$} \\
\hline & & & \multicolumn{5}{|c|}{ Cromosoma 17\%) } & \multicolumn{5}{|c|}{ GEN TP53 (\%) } & \\
\hline & & & $\begin{array}{ll}\overline{0} & \\
\end{array}$ & 1 & 2 & 3 & 4 & 0 & 1 & 2 & 3 & $\geq 4$ & \\
\hline 1 & $\mathrm{~F} / 55$ & Meningioma & 0 & 14 & 86 & 0 & 0 & 6 & 13 & 81 & 0 & 0 & 0,94 \\
\hline 2 & $F / 65$ & Carcinoma de tiroides & 5 & 3 & 64 & 26 & 2 & 4 & 16 & 54 & 24 & 2 & 0,94 \\
\hline 3 & $\mathrm{M} / 60$ & Adenocarcinoma de estómago & 0 & 25 & 66 & 8 & 1 & 11 & 53 & 36 & 0 & 0 & 0,68 \\
\hline 4 & $M / 33$ & Linfoma de Hodgkin & 14 & 30 & 56 & 0 & 0 & 19 & 38 & 41 & 2 & 0 & 0,89 \\
\hline 5 & M/35 & Sarcoma de tejidos blandos & 2 & 93 & 5 & 0 & 0 & 34 & 62 & 4 & 0 & 0 & 0,68 \\
\hline 6 & $\mathrm{M} / 54$ & Adenocarcinoma de colon & 0 & 5 & 91 & 3 & 1 & 3 & 15 & 80 & 1 & 1 & 0,89 \\
\hline 7 & $M / 25$ & Meduloblastoma & 0 & 21 & 79 & 0 & 0 & 0 & 39 & 56 & 5 & 0 & 0,93 \\
\hline 8 & $\mathrm{~F} / 47$ & Adenoma de tiroides & 0 & 5 & 79 & 16 & 0 & 7 & 22 & 69 & 2 & 0 & 0,79 \\
\hline 9 & $\mathrm{M} / 56$ & Carcinoma renal & 0 & 14 & 86 & 0 & 0 & 8 & 32 & 60 & 0 & 0 & 0,82 \\
\hline 10 & $\mathrm{~F} / 77$ & Carcinoma en peritoneo & 0 & 4 & 96 & 0 & 0 & 3 & 27 & 70 & 0 & 0 & 0,85 \\
\hline 11 & $F / 48$ & Carcinoma de cuello uterino & 0 & 2 & 98 & 0 & 0 & 2 & 3 & 95 & 0 & 0 & 0,98 \\
\hline 12 & $\mathrm{M} / 59$ & Carcinoma de lengua & 0 & 13 & 85 & 3 & 0 & 7 & 38 & 55 & 0 & 0 & 0,77 \\
\hline 13 & $F / 69$ & Adenocarcinoma de estómago & 0 & 8 & 89 & 3 & 0 & 6 & 27 & 66 & 1 & 0 & 0,83 \\
\hline 14 & $\mathrm{~F} / 50$ & Sarcoma de tejidos blandos & 0 & 6 & 70 & 17 & 7 & 21 & 26 & 48 & 5 & 0 & 0,60 \\
\hline 15 & $F / 23$ & Poliposis adenomatosa de colon & 0 & 10 & 89 & 1 & 0 & 12 & 44 & 44 & 0 & 0 & 0,69 \\
\hline 16 & $F / 24$ & Astrocitoma cervical & 0 & 0 & 57 & 22 & 21 & 0 & 23 & 69 & 3 & 5 & 0,68 \\
\hline 17 & $M / 39$ & Adenocarcinoma de recto & 0 & 13 & 85 & 2 & 0 & 0 & 32 & 68 & 0 & 0 & 0,89 \\
\hline 18 & $F / 42$ & Mioepitelioma de parótida & 0 & 2 & 98 & 0 & 0 & 0 & 7 & 93 & 0 & 0 & 0,98 \\
\hline 19 & $\mathrm{M} / 66$ & Carcinoma de mandíbula & 0 & 10 & 90 & 0 & 0 & 0 & 48 & 52 & 0 & 0 & 0,80 \\
\hline 20 & $\mathrm{~F} / 73$ & Carcinoma broncogénico & 7 & 3 & 89 & 1 & 0 & 9 & 32 & 58 & 1 & 0 & 0,82 \\
\hline 21 & $\mathrm{M} / 57$ & Carcinoma broncogénico & 5 & 19 & 69 & 7 & 0 & 10 & 59 & 31 & 0 & 0 & 0,68 \\
\hline 22 & $\mathrm{~F} / 46$ & Liposarcoma de tejidos blandos & 0 & 30 & 58 & 8 & 4 & 14 & 48 & 38 & 0 & 0 & 0,67 \\
\hline 23 & $M / 51$ & Carcinoma renal & 0 & 9 & 90 & 1 & 0 & 11 & 25 & 64 & 0 & 0 & 0,80 \\
\hline 24 & $F / 48$ & Carcinoma papilar de tiroides & 1 & 19 & 79 & 1 & 0 & 6 & 34 & 60 & 0 & 0 & 0,86 \\
\hline 25 & $\mathrm{M} / 70$ & Adenocarcinoma de colon & 0 & 27 & 61 & 2 & 10 & 9 & 53 & 28 & 2 & 8 & 0,74 \\
\hline 26 & $F / 29$ & Adenocarcinoma papilar de tiroides & 0 & 28 & 71 & 1 & 0 & 0 & 67 & 33 & 0 & 0 & 0,77 \\
\hline 27 & $\mathrm{~F} / 76$ & Adenocarcinoma de duodeno & 0 & 8 & 92 & 0 & 0 & 6 & 45 & 49 & 0 & 0 & 0,75 \\
\hline 28 & $F / 58$ & Carcinoma de mama & 2 & 16 & 78 & 4 & 0 & 7 & 27 & 62 & 3 & 1 & 0,89 \\
\hline 29 & $\mathrm{M} / 45$ & Carcinoma de laringe & 0 & 7 & 93 & 0 & 0 & 0 & 17 & 83 & 0 & 0 & 0,95 \\
\hline 30 & $\mathrm{M} / 57$ & Carcinoma de cavidad oral & 0 & 0 & 14 & 11 & 75 & 5 & 30 & 39 & 16 & 10 & 0,54 \\
\hline 31 & $F / 57$ & Adenocarcinoma de estómago & 0 & 1 & 98 & 0 & 1 & 23 & 3 & 73 & 0 & 1 & 0,75 \\
\hline 32 & $\mathrm{M} / 67$ & Tumor fusocelular de estómago & 0 & 0 & 98 & 1 & 1 & 53 & 3 & 43 & 0 & 1 & 0,46 \\
\hline 33 & $F / 68$ & Adenocarcinoma de estómago & 0 & 8 & 92 & 0 & 0 & 8 & 23 & 69 & 0 & 0 & 0,84 \\
\hline 34 & $\mathrm{M} / 75$ & Adenocarcinoma de esófago & 0 & 6 & 94 & 0 & 0 & 78 & 8 & 14 & 0 & 0 & 0,19 \\
\hline 35 & $\mathrm{M} / 72$ & Carcinoma de esófago & 0 & 1 & 99 & 0 & 0 & 55 & 5 & 40 & 0 & 0 & 0,43 \\
\hline 36 & $F / 23$ & Adenocarcinoma de colon & 0 & 1 & 99 & 0 & 0 & 16 & 10 & 74 & 0 & 0 & 0,79 \\
\hline 37 & $\mathrm{M} / 63$ & Lipoma de colon & 0 & 1 & 95 & 2 & 2 & 0 & 0 & 93 & 5 & 2 & 1,02 \\
\hline 38 & $\mathrm{M} / 78$ & Adenocarcinoma de colon & 0 & 6 & 93 & 1 & 0 & 4 & 71 & 25 & 0 & 0 & 0,62 \\
\hline \multirow{2}{*}{\multicolumn{3}{|c|}{$\begin{array}{l}\text { Promedio de casos } \\
\text { Promedio de controles }\end{array}$}} & 0,95 & 12,1 & 80 & 3,7 & 3,2 & 12,0 & 29,5 & 55,6 & 1,8 & 0,8 & 0,77 \\
\hline & & & 0,2 & 3,4 & 95,0 & 1,4 & 0 & 1,6 & 6,6 & 89,6 & 2,2 & 0 & 0,97 \\
\hline
\end{tabular}

presentó el mayor porcentaje de núcleos con una señal para el cromosoma 17 (93\%; monosomía).

Otro resultado llamativofue el del caso 30 (carcinoma de la cavidad oral), que mostró $75 \%$ de los núcleos interfásicos con cuatro señales de hibridación para el cromosoma 17 (tetrasómicos) (figura 1). Un resultado similar al anterior se observó en el caso 16 (astrocitoma cervical), el cual presentó un gran porcentaje de núcleos polisómicos; así, 22\% de los núcleos tenían tres señales de hibridación (trisómicos), mientras que $21 \%$ de los núcleos tenían cuatro señales (tetrasómicos).

En el caso. 4 (linfoma de Hodking) se observó el mayor porcentaje (14\%) de núcleos con pérdida de los dos cromosomas 17 (nulisomía); igualmente, tenía un gran porcentaje (30\%) de núcleos con una señal de hibridación (monosómicos).

El caso 2 (carcinoma de tiroides) presentó el mayor porcentaje (26\%) de núcleos con tres señales de hibridación para el cromosoma 17 (trisómicos).

Por otra parte, se encontró deleción del gen TP53 en $89,5 \%(34 / 38)$ de los tumores sólidos examinados (figura 1). Sólo cuatro casos $(10,5 \%)$ no presentaron deleción del gen TP53.

En el grupo de los casos se obtuvo un promedio de $55,6 \%$ para las dos copias del gen TP53, mientras que en el grupo control, el valor promedio fue de $89,6 \%$. Estos valores presentan una diferencia muy importante. De acuerdo con la relación entre 

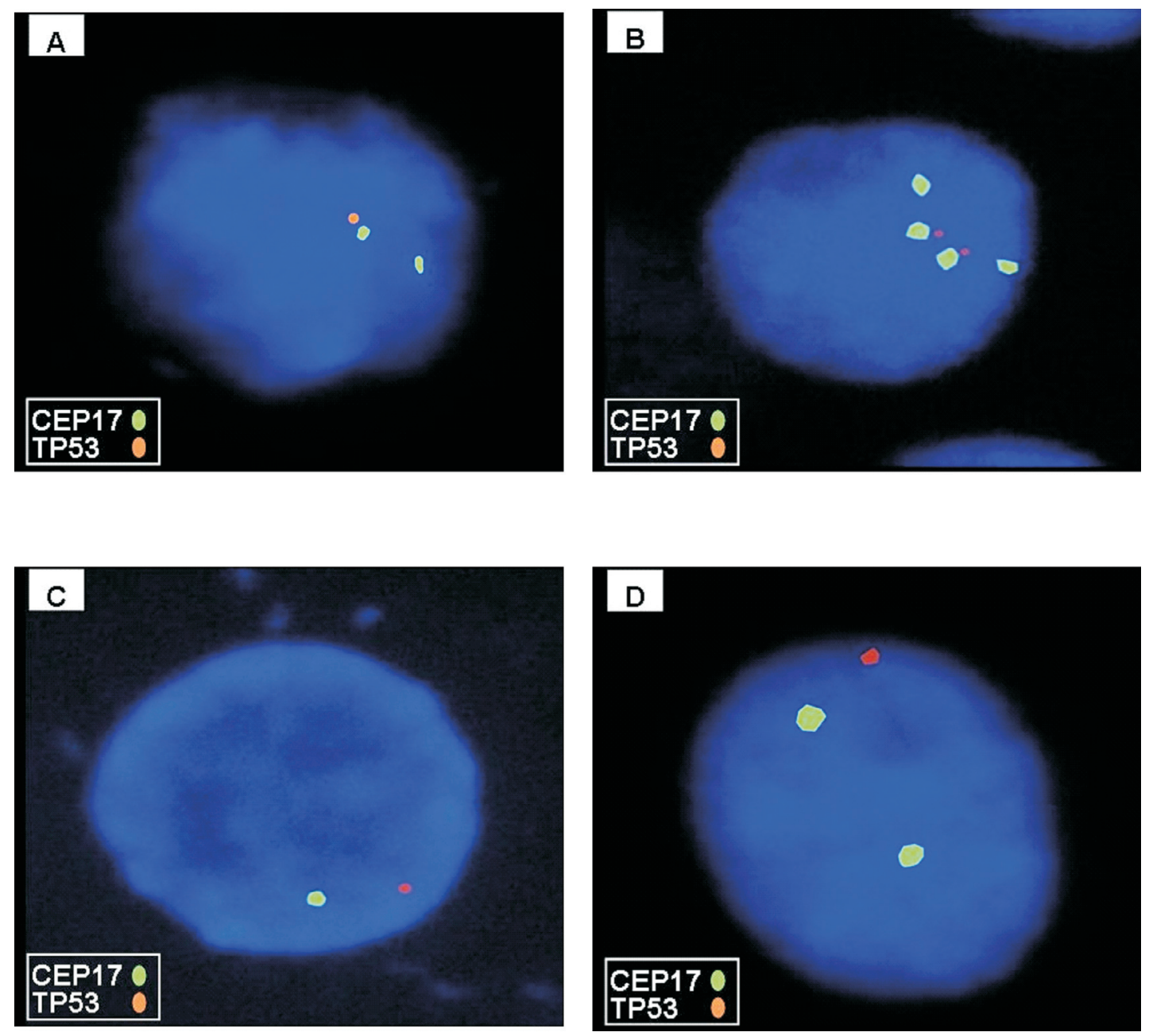

Figura 1. Imágenes de la FISH bicolor mediante sondas CEP17 para el cromosoma 17 (color verde) y LSIp53 para el locus 17p.13.1 del gen TP53 (color naranja) en núcleos interfásicos. Al final de la descripción de cada numeral se informa el resultado de la FISH, siguiendo las recomendaciones del ISCN (An Internacional System for Human Cytogenetic Nomenclature) (2009). A. Carcinoma de laringe, núcleo con dos señales de hibridación para el cromosoma 17 y una señal para el gen TP53 (deleción); nuc ish(D1721x2,TP53×1). B. Carcinoma de la cavidad oral, núcleo con cuatro señales para el cromosoma 17 (tetrasomía) y dos señales para el gen TP53; nuc ish(D1721×4,TP53x2). C. Adenocarcinoma gástrico, núcleo con una señal para el cromosoma 17 (monosomía) y una señal para el gen TP53 (deleción); nuc ish(D1721,TP53)x1). D. Poliposis adenomatosa de colon, núcleo con dos señales para el cromosoma 17 y una señal para el gen TP53 (deleción); nuc ish(D1721x2,TP53×1).

el número total de señales para el gen TP53 y el número total de señales del cromosoma 17 para definir la deleción, se encontró en el grupo de los casos un valor promedio de 0,77 mientras que en el grupo control fue de 0,97.

Estos resultados presentan una diferencia relevante. El caso 34 presentó el valor más bajo para la deleción $(0,19)$, seguido del caso 35 $(0,43)$; ambos eran carcinomas de esófago. Por el contrario, el caso 37 (lipoma de colon) mostró el mayor valor para esta relación $(1,02)$. Similar a lo encontrado para las aneuploidías del cromosoma 17, en el análisis de la FISH bicolor para el gen
TP53, se detectaron diferentes subpoblaciones de núcleos con clones heterogéneos.

Nuevamente, el caso 5 presentó un porcentaje muy bajo (4\%) de núcleos con dos copias para el gen TP53 y, a su vez, tenía un alto porcentaje (62\%) de núcleos con pérdida de una copia del gen TP53 (deleción monoalélica), así como 34\% de los núcleos con pérdida de las dos copia del gen TP53 (deleción bialélica). El caso 26 (adenocarcinoma de tiroides) mostró el mayor porcentaje $(67 \%)$ de núcleos con una señal de hibridación para el gen TP53, mientras que el caso 34 presentó el mayor porcentaje $(78 \%)$ de núcleos con pérdida de las 
dos copias del gen TP53. Por otro lado, el caso 2 presentó el mayor porcentaje (24\%) de núcleos con tres señales de hibridación.

En este trabajo se encontró que la mayoría de las muestras de los tumores sólidos (30/38) tenían subpoblaciones de núcleos con pérdida de las dos copias del gen TP53. Se resalta que cinco casos $(31,32,34,35$ y 36$)$ presentaron altos porcentajes de núcleos con pérdida de las dos copias del gen TP53 (con un rango entre 16\% y 78\%). Dichas muestras correspondían a dos casos de cáncer de estómago, dos de esófago y uno de colon (paciente de 23 años). Sólo en ocho casos se observaron tres o cuatro señales de hibridación para el gen TP53. En general, el análisis de la FISH bicolor para la región 17p13.1, reveló que la pérdida de una copia del gen TP53 fue lo predominante en la mayoría de los casos (figura 1).

Todos los casos que presentaron monosomía del cromosoma 17, también presentaron una sola copia del gen TP53. Por otra parte, de los 14 casos que tenían dos copias del cromosoma 17 (sin aneuploidías), 10 mostraron deleción del gen TP53. Por lo tanto, de las 38 muestras de tumores sólidos analizadas, sólo cuatro casos (10,5\%) (11, 18, 29 y 37) fueron normales para el número de copias del cromosoma 17 y del gen TP53. Estos casos correspondieron a dos tumores benignos y dos carcinomas.

Por otro lado, 15 de las 38 muestras de tumores sólidos eran del sistema gastrointestinal (esófago, estómago, colon, recto y duodeno). Un análisis independiente de este grupo de tumores mostró que el porcentaje de aneuploidía del cromosoma 17 fue de $33,3 \%$ (5/15) y de $93,3 \%$ (14/15) para la deleción del gen TP53 (figura 1).

\section{Discusión}

La detección de alteraciones cromosómicas en los tumores sólidos mediante la técnica de FISH, se ha venido haciendo de forma intensa desde la última década, hasta el punto de convertirse en una técnica de rutina para el análisis citogenético del cáncer.

El estudio citogenético con FISH, similar a lo que ocurre con la citogenética convencional, presenta algunas dificultades técnicas, como la deficiente disociación celular que se tiene en algunos tumores sólidos, debido a la cantidad de tejido conjuntivo y adiposo; otros tumores, como los del sistema gastrointestinal, por su consistencia o viscosidad, se consideran dificultosos para el procesamiento y obtención de núcleos interfásicos. Por esta razón, los núcleos no quedan expuestos y las sondas no se unen a la secuencia complementaria $(2,13)$.

En este estudio, de acuerdo con el diagnóstico histopatológico, es importante resaltar que la mayoría de los tumores examinados fueron malignos. Asimismo, llama la atención que, al momento del estudio, el $37 \%$ de las muestras procedían de individuos menores de 40 años (especialmente, cuatro casos entre 23 y 25 años, todos con tumores malignos); en estos individuos podría suponerse que tendrían un mal pronóstico de la enfermedad. Este tipo de estudios podrían ser útiles en la epidemiología del cáncer en nuestras regiones, con el propósito de identificar factores de exposición ambiental asociados con el cáncer.

En este estudio se encontró una alta frecuencia (63\%) de aneuploidías del cromosoma 17 en los diversos tumores sólidos, lo que concuerda con lo publicado en otros trabajos similares realizados con FISH (11,15-18). Sin embargo, debe mencionarse que los estudios muestran una amplia escala de porcentajes de aneuploidías para el cromosoma 17 (entre $10 \%$ y $80 \%$ ). La aneuploidía del cromosoma 17 es muy frecuente en diversos tumores sólidos, tales como mama, sistema gastrointestinal, vejiga, próstata, riñón, cabeza y cuello $(2,4,19,20)$. Estos hallazgos sugieren que esta alteración cromosómica numérica es clave en el origen de diferentes neoplasias.

En este trabajo, la monosomía fue la aneuploidía más frecuente $(75 \%)$, mientras que la trisomía se presentó con menor frecuencia (17\%). Estos resultados están dentro del rango de porcentajes informados en la literatura $(18,21,22)$. Galluci et al. (23) y Gotte et al. (24) encontraron frecuencias de monosomía de $37,5 \%$ y $27 \%$, respectivamente. Varios trabajos muestran que la pérdida del cromosoma 17 ocurre en las primeras etapas del desarrollo del tumor $(17,18,20,22)$. Lo anterior ha permitido sugerir que la monosomía del cromosoma 17 es una alteración común en los tumores sólidos y que podría considerarse como un marcador cromosómico recurrente.

Por otro lado, en el grupo de tumores sólidos del aparato gastrointestinal se encontró una menor frecuencia de aneuploidía del cromosoma 17 $(33,3 \%)$, Este porcentaje contrasta con el obtenido en todas las muestras evaluadas (63\%), lo cual podría explicarse por el tamaño de la muestra. Sin embargo, es similar al encontrado por Risio et al. (25) en 20 muestras de carcinomas colorrectales, 
mientras que Gomyo et al. (26) y Fringes et al. (20), en muestras de tumores gástricos, encontraron una alta frecuencia de trisomía del cromosoma 17. De esta manera, en la literatura se describen dos patrones de alteraciones cromosómicas (uno monosómico y otro trisómico) en tumores gastrointestinales. Estos trabajos también correlacionan la pérdida del cromosoma 17 con tumores malignos, similar a lo observado en los carcinomas de colon $(15,16,26)$.

En resumen, nuestros resultados muestran que la monosomía del cromosoma 17 fue la alteración numérica más frecuente en todas las muestras de tumores analizadas.

En la década de 1900, Boveri (27) propuso la teoría de la aneuploidía como causa del cáncer y de la inestabilidad cromosómica. Dicha teoría ha sido corroborada en numerosos estudios citogenéticos de tumores sólidos. Los recientes trabajos de Duesberg et al. $(28,29)$ reevalúan los postulados de Boveri, utilizando modelos in vitro con células transformadas, en los que se ratifica que el cáncer se origina por aneuploidías (pérdida o ganancia de cromosomas) y que la inestabilidad cromosómica es proporcional al grado de la aneuploidía y al número de cromosomas afectados $(6,29)$. En conclusión, Duesberg confirma que las alteraciones cromosómicas juegan un papel fundamental en la iniciación y progresión del cáncer, y que, además, los cromosomas de las células cancerosas son inestables por causa de la aneuploidía.

La aneuploidía y la inestabilidad cromosómica afectan la expresión de diferentes genes responsables de la segregación cromosómica, control del ciclo celular, proliferación, síntesis y reparación del ADN (3,4,30-32). Debe anotarse que en el cromosoma 17 se localizan genes importantes implicados en múltiples mecanismos biológicos, como son protooncogenes, genes supresores de tumores, factores de crecimiento, receptores de hormonas, genes de reparación y de estabilidad genómica, entre muchos otros $(1,2,4,7,8)$. Entre los genes que se encuentran en el cromosoma 17, están el ERBB2, BRCA1, EGFR, NME1, THRA, HER2/neu, TP53, TOP2A y NF1 $(1,7,9,17,33)$.

El caso 28 (carcinoma de mama) es interesante porque presentó monosomía del cromosoma 17, considerada una alteración cromosómica común durante el desarrollo de esta neoplasia $(17,34)$. Es bien conocido que los genes BRCA1, HER2/ neu y el TP53 están asociados con la génesis del cáncer de mama $(1,7,9,32)$. En este caso se podría establecer una correlación entre la pérdida de estos genes y el desarrollo del tumor.

En conclusión, la aneuploidía del cromosoma 17 afecta notablemente la expresión de múltiples genes, estableciéndose una vía molecular relacionada con el origen de diversos tumores, como se ha observado en el de mama, colorrectal, ovario, vejiga, cabeza y cuello $(4,8,9,10,20,22,26)$.

Por otra parte, en este estudio se demostró que $89,5 \%$ de los tumores sólidos examinados tenía deleción del gen TP53. Este porcentaje es muy alto comparado con los publicados por otros autores en diferentes tumores sólidos (25,35-38); sin embargo, está dentro del rango informado y concuerda con lo descrito en la literatura en lo referente a que este gen se encuentra alterado en cerca de $50 \%$ de todos los canceres en humanos $(7,9,10,16,22)$.

Nuestros hallazgos demuestran que la deleción del gen TP53 fue predominante en muestras de tumores malignos. Los resultados también corroboran lo informado en cuanto a que la deleción del locus 17p.13.1 ocurre con mayor frecuencia en carcinomas, especialmente en los colorrectales $(25,26,35)$. Además, la deleción se asocia con un mal pronóstico y con una respuesta ineficiente al tratamiento con ciertos medicamentos antineoplásicos $(9,10,39)$. Por lo tanto, la deleción del gen TP53 se considera como una alteración de graves consecuencias para la célula, dada las múltiples funciones biológicas en que está involucrado este gen, específicamente en las del control del ciclo celular, reparación, apoptosis y estabilidad genómica. También se sugiere que la deleción del TP53 juega un papel clave durante la transición de una neoplasia benigna hacia una maligna. No obstante, la utilidad clínica de la deleción del gen TP53 en algunos tumores aún no está bien definida.

En el grupo de tumores del aparato gastrointestinal, la deleción del gen TP53 también presentó una gran frecuencia $(93,3 \%)$, similar a la encontrada en todos los tumores sólidos evaluados. Esta frecuencia informada es notablemente mayor a las publicadas por Fahmy et al. (40) (40\%), Gomyo et al. (26) (77\%) y Risio et al. (25) (60\%). De estos estudios se sugiere que la deleción del gen TP53es una alteración muy frecuente en la carcinogénesis gástrica y colorrectal.

En este estudio se observó una correlación entre la monosomía del cromosoma 17 y la pérdida de una copia del gen TP53. Se ha observado en los 
casos que presentan deleción del TP53 en uno de los dos cromosomas (deleción monoalélica) que, con frecuencia, se presentan mutaciones en el gen TP53 del cromosoma homólogo, lo que conduce a la inactivación del gen $(7,8,26,35,37)$. Por tal razón, la monosomía de cromosoma 17 o la deleción del locus 17p13.1 originarían un estado de "haploinsuficiencia" del gen $\operatorname{TP53}(7,8)$, mientras que, en las muestras que no tienen aneuploidías pero sí la pérdida de las dos copias de gen TP53 (deleción bialélica), no habría expresión del gen. Lo anterior podría explicarse por la teoría de los dos golpes propuesta por Knudson (41).

Por otro lado, en los casos normales de este estudio no podrían descartarse mutaciones en un sólo alelo o en los dos alelos del TP53, puesto que la FISH no detecta mutaciones puntuales. La deleción del TP53 no sólo conduce a la pérdida del control del ciclo celular, sino también, a la acumulación de otros tipos de alteraciones genéticas en las células neoplásicas.

En este estudio realizado con la técnica FISH en núcleos interfásicos, se demostró la presencia de diferentes patrones de señales de hibridación en un mismo tejido. Es decir, se detectaron clones con subpoblaciones de núcleos heterogéneos: monosómicos, disómicos, trisómicos y, en menor frecuencia, tetrasómicos. Estos hallazgos concuerdan con los publicados previamente en la literatura $(16,25,26,35)$. Lo anterior podría explicarse porque en algunas ocasiones las muestras también tumorales incluyen tejido normal o por la heterogeneidad genética intratumoral que caracteriza al tejido neoplásico durante la carcinogénesis $(32,42,43)$. Este mecanismo y la expansión clonal se originan por la inestabilidad cromosómica y por mutaciones en diversos genes como el TP53 $(44,45)$. La heterogeneidad genética se ha evidenciado ampliamente en la mayoría de tumores sólidos y se relaciona con el fenotipo tumoral, la metástasis y la resistencia a determinados medicamentos antineoplásicos. $(32,42,46)$. Por lo tanto, otra de las ventajas que se tienen con la FISH es que permite evaluar la heterogeneidad genética en núcleos individuales, lo que no es posible con otras técnicas moleculares.

Por último, en las cuatro muestras con resultados normales para el número de copias del cromosoma 17 y del gen TP53, no se podría excluir la presencia de otro tipo de alteraciones (cromosómicas o génicas) no evaluadas. Muchos de los tumores sólidos evaluados también se asocian con anomalías en los cromosomas 1, 5, 7, 8, 9, 13 y 18 , entre otros, así como con mutaciones en los genes p21, p16, RB, K-RAS, PTEN, MLH1, DCC, $A P C$ y $C-M Y C(1,2,4,47-49)$. Por tal razón, se necesitan futuras investigaciones que empleen simultáneamente diversas técnicas moleculares, como también, otras técnicas variantes de la FISH, como la SKY-FISH o el CGH-array, para evaluar todos los cromosomas de las células tumorales.

El presente trabajo es el primero en nuestro medio que informa los hallazgos de aneuploidías del cromosoma 17 y deleción del gen TP53 en una variedad de tumores sólidos primarios, empleando la técnica de la FISH bicolor. Los resultados obtenidos aportan información básica sobre la genética de los tumores sólidos; sin embargo, es necesario realizar otros estudios con el propósito de corroborar los resultados informados. Asimismo, es importante ejecutar estudios de epidemiología genética para identificar factores de exposición ambiental a los que está sometida la población colombiana y su relación con la aparición del cáncer.

En conclusión, este estudio confirma que la deleción del gen TP53 y la aneuploidía del cromosoma 17 son alteraciones citogenéticas frecuentes en los tumores sólidos de pacientes colombianos.

\section{Agradecimientos}

Agradecemos la participación de los pacientes en este estudio. A los cirujanos, enfermeras y jefes de los Departamentos de Cirugía y Patología de la Universidad de Antioquia y del Hospital Universitario San Vicente de Paúl de Medellín por su valiosa colaboración. A Enoc Ahumada Rodríguez, por su asesoría en los diagnósticos histopatológicos.

\section{Conflicto de intereses}

Los autores declaramos que no existe conflicto de intereses.

\section{Financiación}

Este trabajo fue financiado por la Universidad de Antioquia, proyecto CPT-0118.

\section{Referencias}

1. Croce CM. Oncogenes and cancer. $N$ Engl $J$ Med. 2008;358:502-11.

2. Albertson DG, Collins C, McCormick F, Gray JW. Chromosome aberrations in solid tumors. Nat Genet. 2003;34:369-76.

3. Weaver BA, Cleveland DW. Does aneuploidy cause cancer? Curr Opin Cell Biol. 2006;18:658-67. 
4. Fröhling S, Döhner H. Chromosomal abnormalities in cancer. N Engl J Med. 2008;359:722-34.

5. Duesberg P, Li R, Fabarius A, Hehlmann R. Aneuploidy and cancer: from correlation to causation. Contrib Microbiol. 2006;13:16-44

6. Duesberg P, Rausch C, Rasnick D, Hehlmann R. Genetic instability of cancer cells is proportional to their degree of aneuploidy. Proc Natl Acad Sci USA. 1998;95:13692-7.

7. Strano S, Dell'Orso S, Di Agostino S, Fontemaggi G, Sacchi A, Blandino G. Mutant p53: an oncogenic transcription factor. Oncogene. 2007;26:2212-9.

8. Herrera JC, Vásquez G, Ramírez JL, Muñetón CM. Papel del gen TP53 en la oncogénesis. Salud UIS. 2004;26:88-99.

9. Petitjean A, Marcel V, Pétré A, Mounawar M, Plymoth A, de Fromentel CC, et al. Recent advances in p53 research: an interdisciplinary perspective. Cancer Gene Ther. 2009; $16: 1-12$

10. Petitjean A, Achatz MI, Borresen-Dale AL, Hainaut $P$, Olivier M. TP53 mutations in human cancers: functional selection and impact on cancer prognosis and outcomes. Oncogene. 2007;26:2157-65.

11. Ramírez GC, Herrera JC, Muñetón CM, Márquez JR, Isaza LF. Análisis de las aneuploidías del cromosoma 17 y deleción del gen TP53 en tumores gastrointestinales por FISH-bicolor. Rev Col Gastroenterol. 2008;23:333-42.

12. Herrera JC, Ramírez GC, Muñetón CM. Estudio de las aneuploidías del cromosoma 17 y la deleción del gen TP53 en neoplasias hematológicas, por la técnica del FISHbicolor. latreia. 2008;21:364-74.

13. Levsky JM, Singer RH. Fluorescence in situ hybridization: past, present and future. J Cell Scl. 2003;116:2833-8.

14. James L, Varley J. Advances in cytogenetic analysis of solid tumours. Cromosome Res. 1996:4:479-85.

15. Rao PH, Mathew S, Lauwers G, Rodríguez E, Kelsen DP, Chaganti RS. Interphase cytogenetics of gastric and esophageal adenocarcinomas. Diagn Mol Pathol. 1993;2:264-8.

16. Kawai T, Hiroi S, Nakanishi K, Sakurai Y, Torikata C. Abnormalities in chromosome 17 and p53 in lung carcinoma cells detected by fluorescence in situ hybridization. Pathol Int. 2004;54:413-9.

17. Watters AD, Going JJ, Cooke TG, Bartlett JM. Chromosome 17 aneusomy is associated with poor prognostic factors in invasive breast carcinoma. Breast Cancer Res Treat. 2003;77:109-14.

18. Frau DV, Lai ML, Caria P, Dettori T, Coni P, Faa G, et al. Trisomy 17 as a marker for a subset of noninvasive thyroid nodules with focal features of papillary carcinoma: cytogenetic and molecular analysis of 62 cases and correlation with histological findings. J Clin Endocrinol Metab. 2008;93:177-81.

19. Bergamo NA, da Silva Veiga LC, dos Reis PP, Nishimoto IN, Magrin J, Kowalski LP, et al. Classic and molecular cytogenetic analyses reveal chromosomal gains and losses correlated with survival in head and neck cancer patients. Clin Cancer Res. 2005;11:621-31.

20. Fringes B, Mayhew TM, Reith A, Gates J, Ward DC. Numerical aberrations of chromosomes 1 and 17 correlate with tumor site in human gastric carcinoma of the diffuse and intestinal types. Fluorescence in situ hybridization analysis on gastric biopsies. Lab Invest. 2000;80:1501-8.

21. Hes O, Síma R, Nemcová J, Hora M, Bulimbasic S, Kazakov DV, et al. End-stage kidney disease: gains of chromosomes 7 and 17 and loss of $Y$ chromosome in nonneoplastic tissue. Virchows Arch. 2008;453:313-9.

22. Hardisson D, Álvarez-Marcos C, Salas-Bustamante A, Alonso-Guervós M, Sastre N, Sampedro A. Numerical aberrations of chromosomes $8,9,11$, and 17 in squamous cell carcinoma of the pharynx and larynx: a fluorescence in situ hybridization and DNA flow cytometric analysis of 50 cases. Oral Oncol. 2004;40:409-17.

23. Gallucci M, Guadagni F, Marzano R, Leonardo C, Merola R, Sentinelli S, et al. Status of the p53, p16, RB1 and HER-2 genes and chromosomes 3, 7, 9, and 17 in advanced bladder cancer: correlation with adjacent mucosa and pathological parameters. J Clin Pathol. 2005;58:367-71.

24. Gotte K, Schafer C, Riedel F, Arens N, Hormann K. Intratumoral genomic heterogeneity in primary head and neck cancer and corresponding metastases detected by dual-FISH. Oncol Rep. 2004;11:17-23.

25. Risio M, Casorzoa L, Chiecchioa L, De Rosaa G, Rossinib F. Deletions of $17 p$ are associated with transition from early to advanced colorectal cancer. Cancer Genet Cytogenet. 2003;147:44-9.

26. Gomyo Y, Osaki M, Kaibara N, Ito H. Numerical aberrations and point mutation of $p 53$ gene in human gastric intestinal metaplasia and well-differenciated adenocarcinoma: analysis by fluorescence in situ hybridization (FISH) and PCR-SSCP. Int J Cancer. 1996;66:594-9.

27. Boveri T. Zur Frage der Entstehung maligner Tumoren. Jena: Gustar Fischer, Jena; 1914. p. 1-64.

28. Duesberg P, Li R, Rasnick D, Rausch C, Willer A, Kraemer A, et al. Aneuploidy precedes and segregates with chemical carcinogenesis. Cancer Genet Cytogenet. 2000;119:83-93.

29. Li L, McCormack AA, Nicholson JM, Fabarius A, Hehlmann R, Sachs RK, et al. Cancer-causing karyotypes: chromosomal equilibria between stabilizing aneuploidy and stabilizing selection for oncogenic function. Cancer Genet Cytogenet. 2009;188:1-25.

30. Kops GJ, Weaver BA, Cleveland DW. On the road to cancer: aneuploidy and the mitotic checkpoint. Nat Rev Cancer. 2005;5:773-85.

31. Michor F. Chromosomal instability and human cancer. Philos Trans R Soc Lond B Biol Sci. 2005;360:631-5.

32. Lengauer C, Kinzler KW, Vogelstein B. Genetic instability in colorectal cancers. Nature. 1997;386:623-7.

33. Liang Z, Zeng X, Gao J, Wu S, Wang P, Shi X, et al. Analysis of EGFR, HER, and TOP2A gene status and chromosomal polysomy in gastric adenocarcinoma from Chinese patients. BMC Cancer. 2008;8:363-72.

34. Reinholz MM, Bruzek AK, Visscher DW, Lingle WL, Schroeder MJ, Pérez EA, et al. Breast cancer and aneusomy 17: implications for carcinogenesis and therapeutic response. Lancet Oncol. 2009;10:267-77.

35. Takahasi Y, Nagata T, Asai S, Shintaku K, Eguchi T, Ishi $\mathbf{Y}$, et al. Detection aberrations on $17 p$ and gene in 
gastrointestinal cancers by dual (two-color) fluorescence in situ hybridization and GeneChip p53 assay. Cancer Genet Cytogenet. 2000;121:38-43.

36. Pfister S, Remke M, Benner A, Mendrzyk F, Toedt G, Felsberg J. Outcome prediction in pediatric medulloblastoma based on DNA copy-number aberrations of chromosomes $6 q$ and $17 q$ and the MYC and MYCN loci. J Clin Oncol. 2009;27:1627-36.

37. Cesar AC, Borim AA, Caetano A, Cury PM, Silva AE. Aneuploidies, deletion, and overexpression of TP53 gene in intestinal metaplasia of patients without gastric cancer. Cancer Genet Cytogenet. 2004;153:127-32.

38. Veiga L, Bérgamo N, Kowalski L, Rogatto S. Classical and molecular cytogenetic analysis in head and neck squamous cell carcinomas. Genet Mol Biol. 2003;26:121-8.

39. Nahi $\mathbf{H}$, Lehmann $\mathbf{S}$, Bengtzen $\mathrm{S}$, Jansson $\mathbf{M}$, Möllgård L, Paul C, et al. Chromosomal aberrations in 17p predict in vitro drug resistance and short overall survival in acute myeloid leukemia. Leuk Lymphoma. 2008;49:508-16.

40. Fahmy M, Skacel M, Gramlich TL, Brainard JA, Rice TW, Goldblum JR, et al. Chromosomal gains and genomic loss of p53 and p16 genes in Barrett's esophagus detected by fluorescence in situ hybridization of cytology specimens. Mod Pathol. 2004;17:588-96.
41. Knudson AG. Two genetic hits (more or less) to cancer. Nat Rev Cancer. 2001;1:157-62.42. Lengauer C, Kinzler KW, Vogelstein B. Genetic instabilities in human cancers. Nature. 1998;396:643-9.

43. Soussi T, Lozano G. p53 mutation heterogeneity in cancer. Biochem Biophys Res Commun. 2005;331:834-42.

44. Nowak MA, Michor F, Iwasa Y. Genetic instability and clonal expansion. J Theor Biol. 2006;241:26-32.

45. Yoshimura A, Sugihara H, Ling ZQ, Peng DF, Mukaisho $\mathrm{K}$, Fujiyama $\mathbf{Y}$, et al. How wild-type TP53 is inactivated in undifferentiated-type gastric carcinomas: analyses of intratumoral heterogeneity in deletion and mutation of TP53. Pathobiology. 2006;73:40-9.

46. Aguilera A, Gómez-González B. Genome instability: a mechanistic view of its causes and consequences. Nat Rev Genet. 2008;9:204-17.

47. Panani AD. Cytogenetic and molecular aspects of gastric cancer: clinical implications. Cancer Lett. 2008;266:99-115.

48. Montenegro Y, Ramírez-Castro JL, Isaza LF, Bedoya G, Muñetón-Peña CM. Análisis genético en pacientes con cáncer colorrectal. Rev Med Chil. 2006;134:1221-9.

49. Worthley DL, Whitehall VL, Spring KJ, Leggett BA. Colorectal carcinogenesis: road maps to cancer. World J Gastroenterol. 2007;13:3784-91. 